\title{
ARTICLE
}

Cite this: DOI: 10.1039/x0xx00000x

\section{Photoluminescent Nanosensors for Intracellular Detection}

\author{
C. A. Kerr ${ }^{\mathrm{a}}$ and R. de la Rica ${ }^{\mathrm{a}, *}$
}

Received 00th January 2012,

Accepted 00th January 2012

DOI: $10.1039 / x 0 x \times 00000 x$

www.rsc.org/

\begin{abstract}
In this manuscript we review the main strategies for detecting changes in intracellular parameters with photoluminescent nanosensors. Examples of the detection of intracellular $\mathrm{pH}$, ion concentration $\left(\mathrm{Na}^{+}, \mathrm{Ca}^{2+}, \mathrm{Zn}^{2+}, \mathrm{Hg}^{2+}\right.$ ), reactive oxygen species (ROS, e.g. hydrogen peroxide), variations in temperature, changes in RNA levels and the detection of enzymatic activity are described in detail. The utilisation of nanomaterials such as quantum dots, silicon carbide nanoparticles, nanoscale metal-organic frameworks, upconverting nanoparticles, fluorescent nanoclusters, gold nanoparticles, nanodiamonds, dendrimers and polymeric nanoparticles in bioimaging is highlighted. When compared to molecular probes, nanosensors combine high sensitivity and selectivity with low toxicity, which are crucial parameters for sensing in live cells.
\end{abstract}

\section{Introduction}

Intracellular mechanisms of regulation allow cells to adapt to changes in their environment. ${ }^{1,2}$ These mechanisms of regulation are often located at particular organelles, which can be considered as bioreactors specifically assembled for developing a particular cellular function. ${ }^{3-5}$ As such, they often have a specific physicochemical environment perfectly tailored to their function. For example lysosomes are intracellular vesicles that are used by cells to degrade molecules. The $\mathrm{pH}$ inside lysosomes is much lower than the $\mathrm{pH}$ in the cell cytosol in order to facilitate the hydrolysis of biomolecules and cellular debris. $^{6}$

The complexity of cell regulation and the compartmentalisation of cell functions make it challenging to study cellular tasks in native conditions. On the one hand, probes aimed at detecting intracellular parameters must penetrate cells without harming them or altering their metabolic state. On the other hand, intracellular probes are often required to target specific organelles where the particular mechanism of regulation is taking place. This complexity is augmented by the fact that regulation may take place in sub-cellular compartments. Imaging these compartments requires submicrometric resolution, which is difficult to attain with many commonly used techniques.

In this manuscript we review current approaches aimed at using photoluminescent nanosensors for the intracellular detection of $\mathrm{pH}$, ions, reactive oxygen species, temperature and RNA. Nanoparticle-based detection schemes have the potential to enable ultrasensitive detections thanks to the outstanding physical properties originating from the nanoscale dimensions of nanomaterials. ${ }^{7-11}$ Furthermore, the yield of cellular uptake of nanomaterials is usually higher than that of molecular probes. ${ }^{12-14}$ And when these molecular probes are encapsulated in nanomaterials they may show reduced toxicity, which is essential to avoid perturbing native cellular conditions. ${ }^{15}$ Compared to biomolecular probes such as green fluorescent proteins (GFPs), nanosensors do not require transfecting cells, which is tedious and cannot be performed in all cell types. ${ }^{16}$ However it should be noted that some nanomaterials may be intrinsically toxic to cells (e.g. shedding of heavy metal ions by some nanoparticles), ${ }^{17}$ or may never escape lysosomes, which is required for the intracellular detection of certain analytes such as RNA. ${ }^{18}$ All in all, the reduced size and outstanding properties of nanosensors make them ideal for non-invasive intracellular detection in real time and in live cells as long as the nanomaterials themselves are not toxic to the cells. The potential cytotoxic effects of nanomaterials have been reviewed elsewhere ${ }^{18-21}$ the reader is referred to this work for a comprehensive review of the subject. The present manuscript is focused on the utilisation of photoluminescent probes for intracellular detection. Other detection strategies such as surface-enhanced Raman spectroscopy (SERS) have been reviewed elsewhere. $^{22}$

\section{Intracellular detection of $\mathbf{p H}$}

Many critical cellular functions such as ionic homeostasis, balance of reactive oxygen species, apoptosis, cell cycle progression and cellular mobility are influenced by the 
intracellular $\mathrm{pH}^{23}$ While the $\mathrm{pH}$ in the cytosol and the nucleus of healthy cells is in the range between 7.2 and 7.4, the secretory and the endocytic pathways may show lower $\mathrm{pH}$, and the $\mathrm{pH}$ inside endosomes and lysosomes is usually in the range between 4.0 and 5.5. ${ }^{24}$ Variations in intracellular $\mathrm{pH}$ can lead to cellular malfunction, which can, in turn, lead to a diseased state. For instance it is well established that cancer cells have abnormal $\mathrm{pH}$, and that cellular acidosis can trigger the early phase of apoptosis and lead to DNA fragmentation. ${ }^{25}$

Traditional methods for detecting intracellular $\mathrm{pH}$ include microelectrodes and fiber optic sensors. ${ }^{26}$ However these methods are too invasive and create toxicity due to damage to the cell membrane when entering the cell. Furthermore, they occupy a large volume within the cell, and therefore they cannot be used to detect $\mathrm{pH}$ changes in sub-cellular compartments. One of the most promising techniques for overcoming the issues associated to the utilisation of microsensors consists in using fluorescent molecular probes ${ }^{27}$ and fluorescent proteins (e.g. GFPs) ${ }^{28}$ as $\mathrm{pH}$ sensors. The intensity of the fluorescence emission of these probes changes depending on the environmental $\mathrm{pH}$, which is the basis for the utilisation of fluorescent molecules as $\mathrm{pH}$ sensors. These methods yield a fast response that can be easily quantified using fluorescence microscopy or FACS (fluorescence-activated cell sorting), which are techniques that are commonly found in biology laboratories. However free fluorescent dyes still have complications related to cell delivery and toxicity, ${ }^{29}$ and the fluorescence emission can easily fluctuate due to factors unrelated to variations of intracellular $\mathrm{pH}^{30}$ Below we analyse new approaches based on nanosensors that tackle these issues.

False signals originated by fluctuations in the fluorescence emission of the intracellular probes can be avoided by using quantum dot nanosensors and fluorescence lifetime imaging microscopy (FLIM). ${ }^{30}$ Quantum dots (QDs) are photoluminescent semiconducting nanoparticles whose emission is intimately related to the nanoparticle size. ${ }^{17}$ Other common attributes of quantum dots are high quantum yields, resistance to photobleaching, photostability, narrow photoluminescence spectra and broad absorption spectra. ${ }^{31}$ These qualities make them ideal for long-term bioimaging experiments. Furthermore, their photoluminescence decay is notoriously long and they exhibit multiexponential decay kinetics, which makes them perfectly suited for FLIM measurements. $^{30}$

The $\mathrm{pH}$ nanosensors were obtained by capping core/shell $\mathrm{CdSe} / \mathrm{ZnS}$ quantum dots with mercaptopropionic acid (MPAQDs). When the nanosensors were immersed in solutions simulating the intracellular environment at different $\mathrm{pH}$ values their photoluminescence lifetime changed with the $\mathrm{pH}$ accordingly (Fig. 1A). FLIM measurements were able to detect very small changes in fluorescence lifetime (as small as one hundredth of a nanosecond change) indicating the high sensitivity of this technique. When the nanosensors were tested on live cells, FLIM images demonstrated that the QDs were incorporated into the cytoplasm of the cell via endocytosis. The morphology of the cells remained unchanged after the QDs entered proving the low toxicity and easy entry in to the cell. The nanosensors were able to detect variation in intracellular $\mathrm{pH}$ in the range between 5.70 and 8.19 within CHO-k1 cells (Fig. 1B).

Silicon carbide (3C-SiC) nanoparticles are an interesting alternative to CdSe QDs for measuring intracellular $\mathrm{pH}^{32}$ Silicon carbide is wear and corrosion resistant and has little interactions with biological fluids, which makes it a particularly interesting material for bioimaging in vivo. ${ }^{33} 3 \mathrm{C}-\mathrm{SiC}$ nanoparticles show green fluorescent emission arising from surface structures induced by $\mathrm{H}^{+}$and $\mathrm{OH}^{-}$dissociated from water, and therefore is $\mathrm{pH}$ dependent. ${ }^{34}$ It has been found that the response of pure $3 \mathrm{C}-\mathrm{SiC}$ nanoparticles to changes in the intracellular $\mathrm{pH}$ of $\mathrm{HeLa}$ cells is comparable to the $\mathrm{pH}$ sensitivity of a commonly used fluorescent indicator, BCECF, therefore proving that the nanoparticles are capable of measuring the intracellular $\mathrm{pH}$ accurately. ${ }^{32}$ In this work the nanosensors were able to detect a decrease in intracellular $\mathrm{pH}$ as a consequence of inducing apoptosis in the HeLa cells, a result that was corroborated with the BCECF probe.
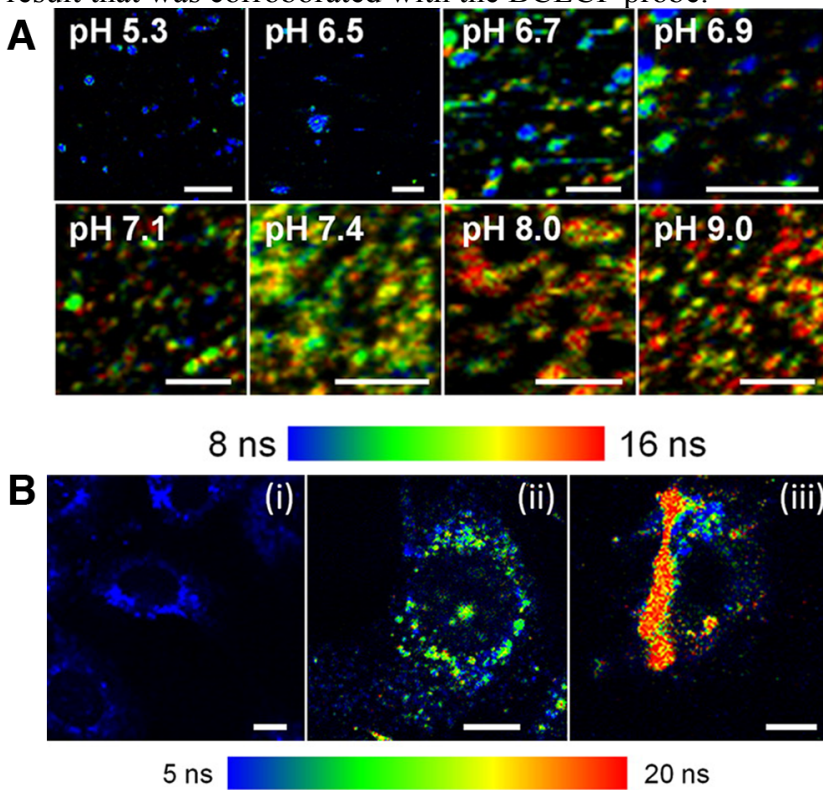

Figure 1. Detection of intracellular $\mathrm{pH}$ with quantum dots (QDs) and fluorescence lifetime imaging microscopy (FLIM); (A) FLIM images of QDs in solutions at different $\mathrm{pH}$ values. Scale bars: $10 \mu \mathrm{m}$; (B) FLIM images of MC3T3-E1 cells before incubation with QDs (i); or after incubation with the nanoprobes at intracellular $\mathrm{pH}$ values of 4.87 (ii) and 8.14 (iii). Reprinted with permission from ${ }^{30}$. Copyright (2013) American Chemical Society.

Nanoscale metal-organic frameworks (NMFOs) modified with fluorescein isothiocyanate (FITC) have also been utilised to measure intracellular $\mathrm{pH}^{35}$ Metal-organic frameworks (MOFs) are porous crystalline structures made up of metal ions and organic molecules held together by strong bonds; MOFs are robust materials that are empty inside. ${ }^{36}$ FITC is an organic fluorescent dye commonly used as a fluorescent label and is known to be sensitive to changes in $\mathrm{pH}$ levels. ${ }^{37}$ The nanosensor was prepared by covalently conjugating UiO (a Zirconium containing MOF) and FITC. ${ }^{35}$ The NMOFs were remarkably insensitive to dye leaching (less than $4.5 \%$ FTIC observed in the supernatant after being incubated in the solvent for 24 hours), which is a common problem associated with the use of fluorescent dyes in other strategies for $\mathrm{pH}$ detection. The nanosensor retained its morphology after 12 hours in the solvent most likely owing to the characteristic mechanical and thermal stability observed in MOFs. Furthermore the large surface area of the NMFOs enabled large amounts of FTIC to be loaded onto their surface, which is an advantage compared to other strategies for encapsulating fluorescent dyes. It was demonstrated that the $\mathrm{pH}$ sensing function of FITC was unaffected after conjugation to $\mathrm{UiO}$ (Fig. 2A). The nanosensors 
were used to study the endosome acidification process in real time. This was accomplished via live cell imaging in which the emission of F-UiO was monitored (Fig. 2B). The experiments demonstrated that the endosomes acidify as they mature in accordance with previously published experiments. ${ }^{38}$ The nanosensors proved to be sensitive and robust, entered the cells rapidly and had the ability to detect the $\mathrm{pH}$ in real time and in live cells without causing dye leaching and without the fluorescent dye losing its sensing properties.

\section{A}

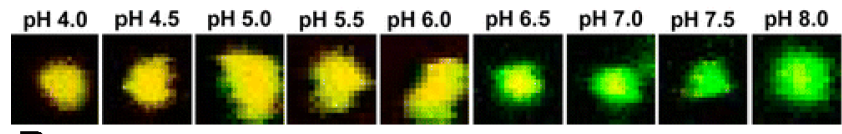

B
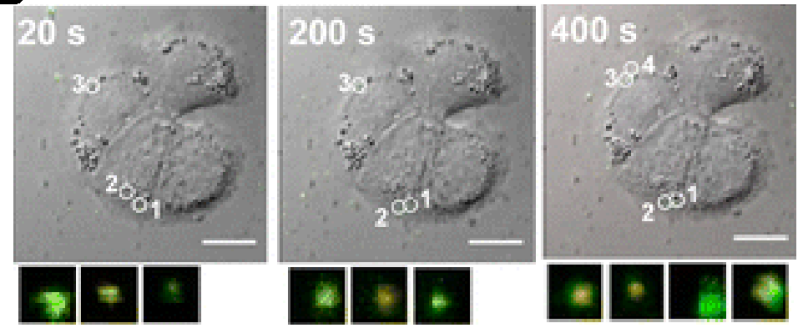

Figure 2. Detection of intracellular $\mathrm{pH}$ with nanoscale metalorganic frameworks (NMOFs); (A) Images showing the fluorescence emission of the nanosensors in buffers with different $\mathrm{pH}$ values; (B) Real time detection of $\mathrm{pH}$ changes in live cells; Scale bar: $10 \mu \mathrm{m}$. Reprinted with permission from 35. Copyright (2014) American Chemical Society.

Upconverting nanoparticles (UPCNs) can convert absorbed light with lower energy, usually in the near-infrared (NIR) range, into photoluminescence emission with higher energy. ${ }^{39}$ Upconversion fluorescence shows reduced autofluorescence backgrounds due to the NIR excitation, which makes it particularly attractive for bioimaging. ${ }^{40}$ This phenomenon was adapted for the intracellular detection of $\mathrm{pH}$ in a nanosensor design incorporating upconversion resonance transfer between nanoparticles and a pH-sensitive fluorophore. ${ }^{41}$ The nanoparticles consisted in hexagonal $\mathrm{NaYF}_{4}: \mathrm{Yb}^{3+}, \mathrm{Er}^{3+}$ crystals with dimensions $28 \times 36 \mathrm{~nm}$ coated with aminosilane. By referencing changes in the emission properties of the dye with respect to the $\mathrm{pH}$ insensitive upconversion of the nanoparticles it was possible to detect changes in $\mathrm{pH}$ in the range between 7.2 and 2.5. The nanoparticles were internalised by cells in endosomes, in which a lower $\mathrm{pH}$ value could be measured.

\section{Intracellular detection of ions}

Metal ions are essential to many cell functions, from the maintenance of the membrane potential ${ }^{42}$ to signalling pathways. ${ }^{43}$ Many enzymes indispensable for the cell life also require metal ions to be catalytically active. However high levels of intracellular ions can be toxic to cells and may even result in cell death. Therefore there is a great interest in developing new probes for detecting the concentration of specific ions in live cells in order to elucidate the role of each metal ion in the different cellular functions.

Calcium ions relay intracellular signals to the cell surface by moving between the cytosol and intracellular stores, and therefore are crucial elements of cell regulation. ${ }^{44}$ Many disorders may arise from abnormal concentrations of intracellular $\mathrm{Ca}^{2+}$ including cardiovascular disease hypertension, and diabetes. ${ }^{45,46}$ In this context a method for detecting intracellular $\mathrm{Ca}^{2+}$ can lead to a better understanding of the associated disorders and potentially to new and improved therapeutics.

Calcium ions can be detected with PEBBLE nanosensors. ${ }^{47}$ PEBBLE stands for probes encapsulated by biologically localised embedding. ${ }^{48}$ The nanosensor is composed of a polyacrylamide nanoparticle matrix that encapsulates a sensing dye (Rhodamine-2) and a reference dye (hilyte fluor 647). A reference dye is added to make ratiometric detection possible, which is less affected by variations in dye concentration, photobleaching, leakage of dye and optical instabilities. When testing the specificity of rhodamine- 2 for $\mathrm{Ca}^{2+}$ the researchers realised that $\mathrm{Mg}^{2+}$ caused the observed $\mathrm{K}_{\mathrm{d}}$ value to increase from $429 \pm 38 \mathrm{nM}$ to $786 \pm 65 \mathrm{nM}$, which means that $\mathrm{Mg}^{2+}$ weakens the affinity of the rhodamine- 2 dye for $\mathrm{Ca}^{2+}$. However if this is taken into account when calibrating the $\mathrm{Kd}$ value then $\mathrm{Ca}^{2+}$ measurements should not be affected. To avoid interference from certain transition-metal ions, a heavy-metalion chelator N,N,N',N'-tetrakis-(2-pyridylmethyl)ethylenediamine was added. When the protein bovine serum albumin (BSA) was added to the PEBBLE nanosensor the affinity for $\mathrm{Ca}^{2+}$ was unaffected. This was expected, as BSA was too large to enter the nanoparticle matrix and therefore could not affect the interaction between rhodamine- 2 and $\mathrm{Ca}^{2+}$. The rhodamine-2 PEBBLES entered 9L glicosarcoma cells by non-specific endocytosis and experienced changes in fluorescence emission related to changes in the intracellular concentration of $\mathrm{Ca}^{2+}$. This was tested by adding a calcium ionophore and changing the extracellular concentration of $\mathrm{Ca}^{2+}$.
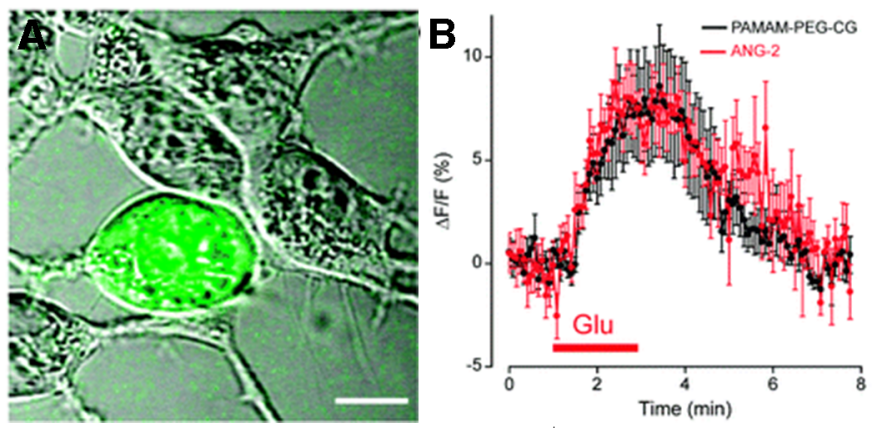

Figure 3. Intracellular detection of $\mathrm{Na}^{+}$with fluorescent dyes encapsulated in dendrimers; (A) Image of HEK-293 cell filled with the nanosensor; Scale bar: $10 \mu \mathrm{m}$. (B) an increase in the intracellular concentration of $\mathrm{Na}^{+}$provoked by glutamate could be detected with the dendrimer nanosensors (black), a result that was corroborated with the $\mathrm{Na}^{+}$dye ANG-2 (red). Reprinted with permission from 49. Copyright (2012) American Chemical Society.

Nanosensors for the detection of intracellular $\mathrm{Na}^{+}$can be fabricated by encapsulating a $\mathrm{Na}^{+}$-responsive probe, CoroNa green $(\mathrm{CG})$, in dendrimers. ${ }^{49}$ Dendrimers are nanomaterials made of branched polymers that can encapsulate small molecules and act as nanocontainers. ${ }^{50}$ In the proposed $\mathrm{Na}^{+}$ nanosensors the encapsulated dyes are remarkably stable with time; one month after their preparation only $1.6 \%$ of the dye is found free in solution. The nanosensors were delivered into live neurons in acute neocortical brain slices by single-cell electroporation. The small size of the probes $(6.57 \pm 0.04 \mathrm{~nm}$ measured by TEM) facilitated imaging small cellular compartments, unlike previous nanosensors which were too large to afford such resolution. ${ }^{51}$ The nanosensors were not 
affected by varying intracellular levels of $\mathrm{K}^{+}, \mathrm{Ca}^{2+}$ or $\mathrm{pH}$. When the nanoprobes were delivered into HEK-293 cells permanently transfected with $\mathrm{Na}^{+}$-coupled glutamate transporter GLT-1, the increase in the concentration of $\mathrm{Na}^{+}$could be detected as an increase in fluorescence emission (Fig. 3A). The observed nanosensors response was identical to the response observed from Asante Natrium Green-2 (ANG-2), a commonly used molecular probe to detect $\mathrm{Na}^{+}$(Fig. 3B).

Intracellular mercury ions can be detected with nanosensors comprising red fluorescent gold nanoclusters (Au NCs) and blue-fluorescent conjugated-oligomer substituted polyhedral oligomeric silsesquioxane (POSSFF). ${ }^{52}$ Nanoclusters refers here to metallic nanoparticles less than $2 \mathrm{~nm}$ in diameter. These ultrasmall fluorescent particles can easily enter cells and are highly biocompatible making them suitable for intracellular detection. ${ }^{53}$ The detection of $\mathrm{Hg}^{2+}$ is based on Förster Resonant Energy Transfer (FRET) between the POSSFF nanodot, which is a perfectly three-dimensional light harvesting molecule, and the gold nanocluster, which is a stable probe that is only slightly affected by ionic strength and $\mathrm{pH}$. The basis of the detection is the strong metalophillic $\mathrm{Hg}^{2+} / \mathrm{Au}^{+}$interaction, which is highly selective and quenches the emission from the red-fluorescent nanoclusters. This results in the observation of the blue fluorescence of the POSSFF nanodot. The nanosensors were internalised by breast cancer cells (MCF-7). When the cells were incubated with $\mathrm{Hg}^{2+}$ ions they showed bright blue fluorescence whilst cells free of $\mathrm{Hg}^{2+}$ showed pink fluorescence (Fig. 4). The proposed $\mathrm{Hg}^{2+}$ nanosensors could be useful in medical applications such as the treatment and diagnosis of diseases caused by mercury poisoning, which is a global environmental problem.
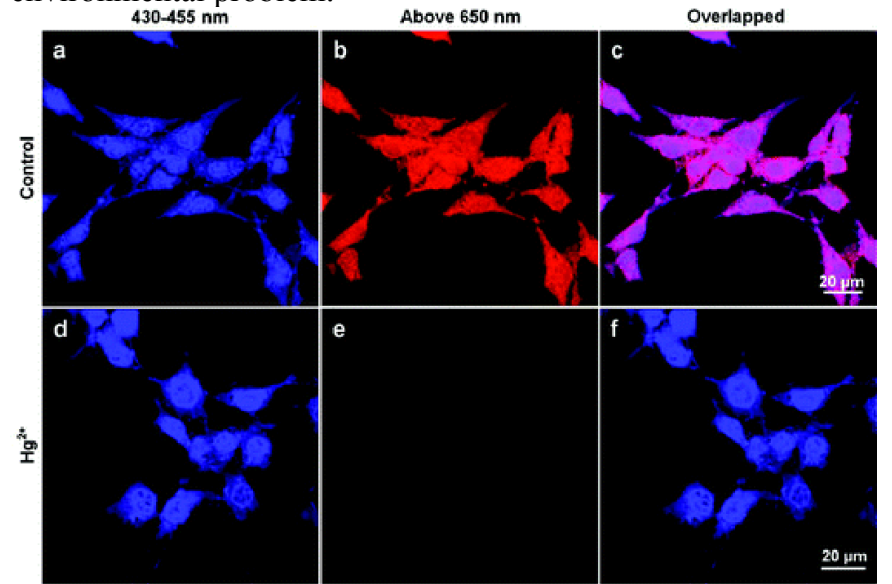

Figure 4. Detection of $\mathrm{Hg}^{2+}$ in MCF-7 cells stained by the POSSFF/R-AuNC nanosensor in the absence $(\mathrm{a}-\mathrm{c})$ or presence (d-f) of $\mathrm{Hg}^{2+}$. Reprinted with permission from 52. Copyright (2012) American Chemical Society.

Zinc plays an important role in the intracellular environment taking part in maintaining many cellular functions such as gene expression, signal transmission and cell growth. ${ }^{54}$ Quinoline probes are commonly used to detect $\mathrm{Zn}^{2+}$ using fluorescence microscopy. However these molecular probes can cause cellular photodamage and autofluorescence thus making them undesirable for live cell detection. ${ }^{55} \mathrm{Zn}^{2+}$ nanosensors were developed by conjugating a quinoline derivative to the surface of carbon nanodots in hopes that the nanoparticles could protect the cellular environment, increase the specificity of the probe and improve the cellular delivery of the sensor. When $\mathrm{Zn}^{2+}$ was added to the solution containing the nanosensor, the quinoline derivatives chelated the ions and an increase in emission in the spectral region between $\sim 440 \mathrm{~nm}$ to $510 \mathrm{~nm}$ was observed, thus confirming that the nanosensor was capable of detecting $\mathrm{Zn}^{2+}$. HeLa cells treated with the proposed nanosensors showed clear green fluorescence upon addition of $\mathrm{ZnCl}_{2}$, which validated the utilisation of the nanosensors for intracellular detection of $\mathrm{Zn}^{2+}$.

\section{Intracellular detection of ROS}
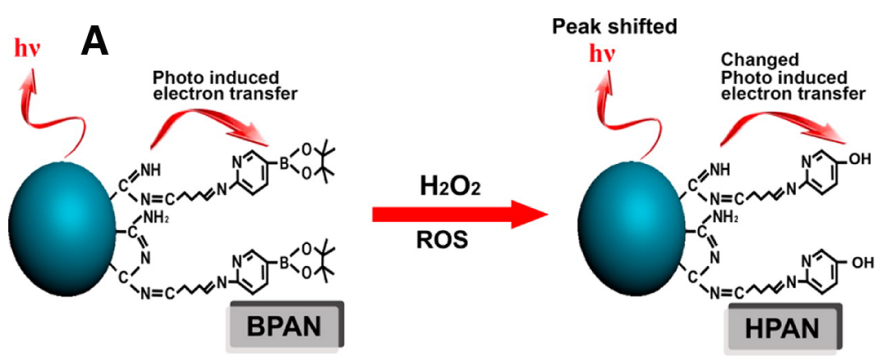

B
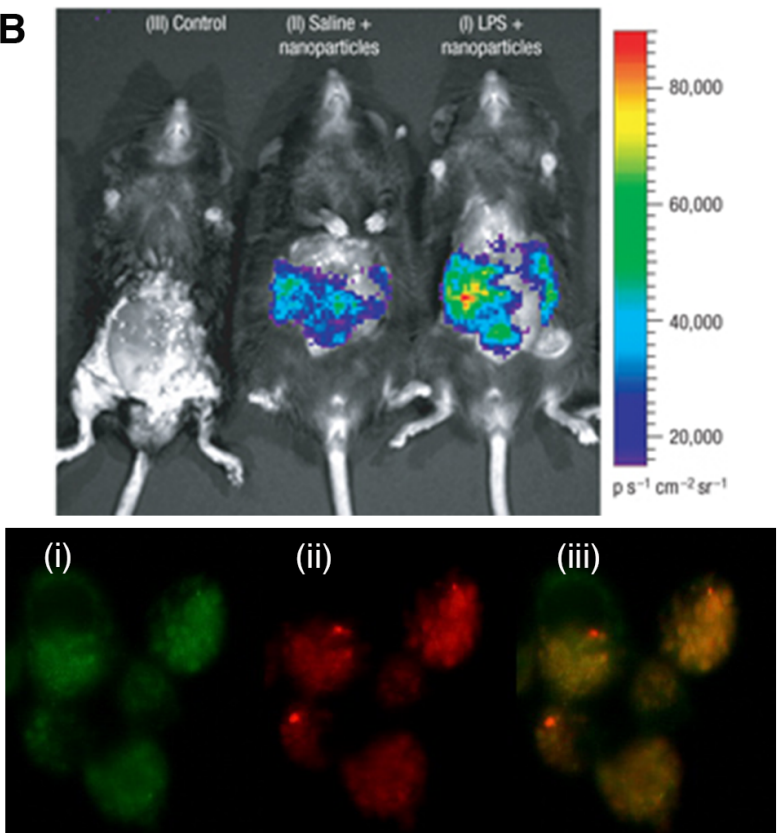

Figure 5. Intracellular detection of reactive oxygen species (ROS); (A) Nanosensor design based on boronate fluorescent dyes (BPAN) and polyacrylonitrile nanoparticles; Reprinted with permission from 58. Copyright (2012) American Chemical Society; (B) Detection of hydrogen peroxide in the peritoneal cavity of mice after injection of peroxalate nanoparticles and after inducing an inflammatory response with lipopolysaccharides (LPS); Reprinted with permission from 59. Copyright (2007) Nature Publishing Group; (C) Detection of ROS in NR8383 cells; Fluorescence images of (i) DHR 123; (ii) Alex Fluor ${ }^{568}$; (iii) Combined images; Reprinted with permission from 48. Copyright (2009) Elsevier.

Reactive oxygen species (ROS) include both oxygen radical species (e.g. superoxide), and non-radical oxygen species (e.g. hydrogen peroxide). They are mainly produced by mitochondria. ${ }^{56}$ Although ROS have been traditionally associated to cell damage, several studies have highlighted their relevance in other aspects of cell regulation such as differentiation and immunity. ${ }^{57}$ Furthermore ROS such as hydrogen peroxide are often overproduced in the early stages of 
some neurodegenerative disorders such as Alzheimer's and Parkinson's disease. ${ }^{58}$ However the exact pathway by which these molecules are produced is still unknown. In this context, a non-invasive method for detecting increased amounts of hydrogen peroxide could potentially be very useful for studying these diseases and finding new therapies in the future.

An efficient method for detecting intracellular hydrogen peroxide consists of using molecular fluorescent probes. ${ }^{60}$ Many of these probes are boronates that are selectively oxidised by hydrogen peroxide in order to produce fluorescent species. However these probes suffer from many limitations including high risk of cytotoxic effects, non-specific binding, low tissue penetration and very slow reaction rates with hydrogen peroxide, which limit real-time measurements. ${ }^{59}$ Some of these limitations can be overcome by substituting molecular probes for polyacrylonitrile nanoparticles modified with boronate fluorescent dyes (BPAN, Fig. 5A). ${ }^{58}$ The detection mechanism involves a photoinduced electron transfer between the boronate and a Schiff base, which results in an increase in fluorescence emission. The BPAN nanoparticles penetrate cells by endocytosis, but are not found in the mitochondria or the cell nucleus. The nanosensors are able to detect the production of hydrogen peroxide in RAW264.7 cells and show negligible cytotoxic side effects.

Peroxalate esters can also be used to fabricate nanosensors aimed at measuring intracellular ROS. ${ }^{59}$ The peroxalate nanoparticles are generated by conjugating a polymer containing a peroxalate ester backbone with the fluorescent dye pentacene. Chemiluminescence is observed when the peroxalate nanoparticles come into contact with hydrogen peroxide even at nanomolar concentrations. The chemiluminescence is originated by the formation of a diaxetanedione bond, which excites the encapsulated fluorescent dyes. The nanosensors are highly specific to hydrogen peroxide over other reactive oxygen species. A great advantage to this method is that the wavelength for chemiluminescence is tuneable in the spectral region between 450 and $630 \mathrm{~nm}$ depending on what dye is used to modify the nanoparticles. The abundance of peroxalate esters gives a continuous energy source meaning the chemiluminescence will occur for an extended period of time in the presence of hydrogen peroxide. It was demonstrated that the nanosensors could detect hydrogen peroxide in vivo in the peritoneal cavity of mice during a lipopolysaccharide-induced inflammatory response (Fig. 5B).

PEBBLE sensors can also be used to detect intracellular hydrogen peroxide. The PEBBLE nanosensors can be obtained by growing a polyacrylamide nanoparticle matrix encapsulating the fluorescent probe dihydrorhodamine 123 (DHR 123). ${ }^{48}$ The sensor is easily delivered into the cell through phagocytosis and does not kill the cells, as proved by a MTT assay. DHR 123 is oxidised by hydrogen peroxide to yield the fluorescent rhodamine 123. Consequently the fluorescence observed is proportional to intracellular hydrogen peroxide. A potential drawback to this method is that DHR 123 is not selective and can react in the presence of other reactive oxygen species. The nanosensors were loaded into NR8383 rat alveolar macrophage cells via phagocytosis. Addition of phorbol-12-myristate-13acetate (PMA) enabled the measuring of the generation of ROS in live cells (Fig. 5C).

\section{Intracellular detection of temperature}

Thermogenesis is vital to life, and studying cellular mechanisms of thermal regulation is a central field in modern biology. ${ }^{61,62}$ This requires mapping temperature changes in subcellular organelles with nanometric resolution, which is an extremely challenging endeavour. ${ }^{63}$ One of the most successful approaches for detecting thermal variations in live cells consists in measuring the temperature-sensitive fluorescence of green fluorescent proteins (GFPs). ${ }^{64}$ However, this approach is limited by its low sensitivity, intrinsic fluctuations in the fluorescence rate and variations in fluorescence originating from the local chemical environment and the optical properties of the surrounding medium. Moreover, methods based in GFPs require cellular transfection, which is laborious and may be problematic in some primary cell types. In this context it would be desirable to fabricate nanosensors that penetrated cells and measured changes in temperature with great accuracy.

Using nanodiamonds as thermal nanosensors is one of the most promising approaches for measuring intracellular temperature with nanometric resolution. ${ }^{63}$ This can be achieved by manipulating the spin states of nitrogen vacancy (NV) centres in nanodiamond materials. Nitrogen vacancy centres are defects present in diamond. The temperature can be detected by analysing the spin frequency associated with the NV centre in a pure diamond bulk. In the absence of an external magnetic field the transition frequency $(\Delta)$ between the $\left|\mathrm{m}_{\mathrm{s}}=0\right|$ and $\left|\mathrm{m}_{\mathrm{s}}= \pm 1\right|$ has a temperature dependence $(\mathrm{d} \Delta / \mathrm{dT}=-2 \pi \mathrm{X} 77 \mathrm{kHz} \mathrm{K}-1)$ thus temperature can be measured from analysing the transition frequency.

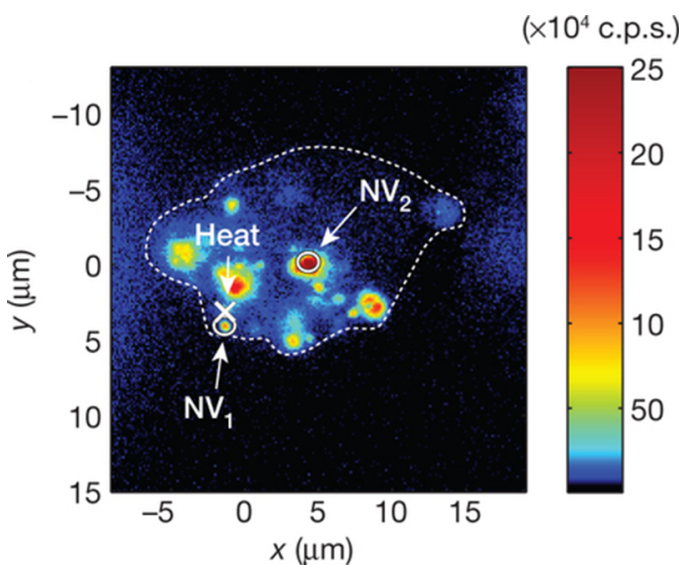

Figure 6. Intracelullar detection of temperature with nanodiamonds; The cross marks the position of the gold nanoparticle used for increasing the temperature; $\mathrm{NV}_{1}$ and $\mathrm{NV}_{2}$ represent the location of the nanodiamonds; The dotted line outlines the cell membrane; Colour bars indicate the fluorescence in counts per seconds (c.p.s). Reprinted with permission from 63. Copyright (2013) Nature Publishing group.

Experiments were carried out using nanodiamonds (with nitrogen vacancy centre defects) combined with gold nanoparticles, which can be easily heated using a laser. The nanodiamonds combined with gold nanoparticles were inserted into WS1 cells to determine if intracellular temperature measurements were feasible. Both nanomaterials were colocalised using a confocal microscope with two independent scanning beams. The cells were probed in two distinct areas $\mathrm{NV}_{1}$ and $\mathrm{NV}_{2}$ whilst locally heating an individual gold nanoparticle (Fig. 6). $\mathrm{NV}_{1}$, closer in distance to the gold 
nanoparticle has stronger temperature dependence as a function of the laser power than $\mathrm{NV}_{2}$ thus proving that controlled temperature differences can be achieved by varying the distances. The readout time was efficiently fast (millisecond time resolution).

Nanodiamonds are biocompatible and robust and can enter the cells fairly easily via nanowire-assisted delivery. The measurements were accurate and fast with specificity towards temperature measurements. A drawback to this technique is that real time measurements are not yet possible.

Fluorescent polymeric thermometers (FPT) can also be used to detect intracellular temperature with great accuracy. ${ }^{62}$ The nanosensors contain a thermoresponsive polymeric unit, a hydrophilic unit and a fluorescent unit. The thermoresponsive unit shrinks when the temperature increases, which results in a stronger fluorescence emission that enables detecting variations in temperature in real time. When combined with fluorescence lifetime imaging this approach enables detecting intracellular changes of temperature happening at particular organelles. For example FPTs were used to detect variations in temperature in the nucleus and centrosome of a COS7 cell. The nanosensors were also used for detecting thermogenesis in mitochondria. These studies revealed that the higher temperatures registered in these subcellular compartments were originated by a local mechanism of heat generation.

\section{Intracellular detection of RNA}

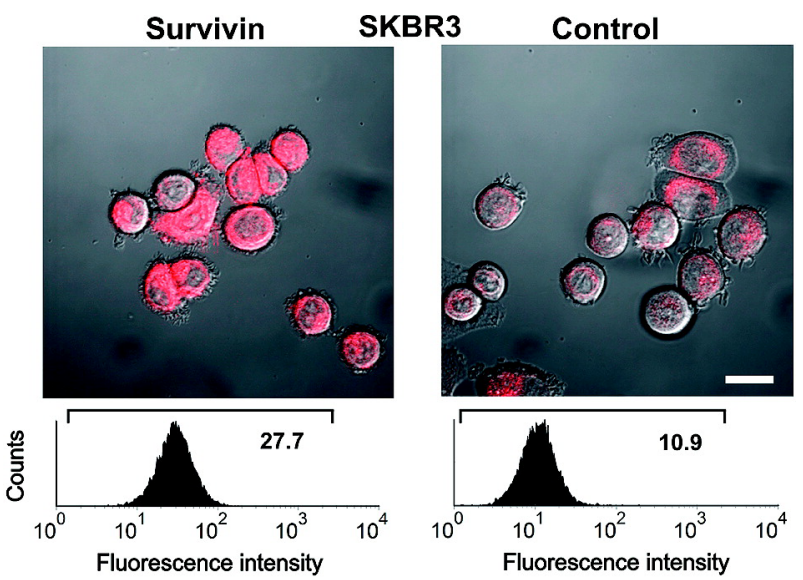

Figure 7. Intracellular detection of survivin mRNA in SKBR3 cells with gold nanoparticles decorated with fluorescent oligonucleotides (nano-flares). Reprinted with permission from 65. Copyright (2007) American Chemical Society.

RT-PCR (reverse transcription polymerase chain reaction) is the most commonly used method for the detection of messenger RNA (mRNA). However RT-PCR requires extracting mRNA from cells, and therefore it is not adequate for measuring changes in mRNA levels in real time. Nanoflares are nanosensors that quantify intracellular mRNA levels within living cells. ${ }^{65}$ The nanosensors consist of gold nanoparticles functionalised with a monolayer of fluorophorelabelled oligonucleotides or flares. The fluorescence of the flare is quenched by the Au NP. However, when the oligonucleotides bind to a target mRNA a flare is displaced from the Au NP surface which results in an observed increase in fluorescence. This enables the detection of a target mRNA in real time (Fig. 7). The released oligonucleotide can be used to transfect the cells. For example it was demonstrated that nanoflares could release siRNAs for knockdown experiments aimed at reducing the intracellular levels of survivin RNA transcripts. A great advantage of nano-flares is that the nanoparticles enter the cells fairly easily and so there is no need for a separate transfection agent or the use of micro-injection methods. Furthermore, nano-flares are non-toxic and have low immunogenicity. The signal can be easily multiplexed by simply modifying nanoparticles with different oligonucleotides labelled with fluorescent probes emitting in different spectral regions, for example Cy5 and Cy3. ${ }^{66}$

Upconversion fluorescent nanoparticles have been proposed as nanosensors for the detection of the intracellular fate of small interference RNA (siRNA). ${ }^{67}$ Silica-coated $\mathrm{NaYF}_{4}$ upconversion nanoparticles codoped with $\mathrm{Yb} / \mathrm{Er}$ were fabricated and modified with a positively charged silane in order to capture negatively charged siRNA. The siRNA was stained with the fluorescent molecule BOBO3. FRET between the upconversion nanoparticle and $\mathrm{BOBO} 3$ resulted in a decrease in fluorescence emission. When the nanoparticles penetrated cells they released siRNA and the fluorescence emission increased. This enabled monitoring the release of siRNA in live cells, which could greatly improve transfection protocols in the future.

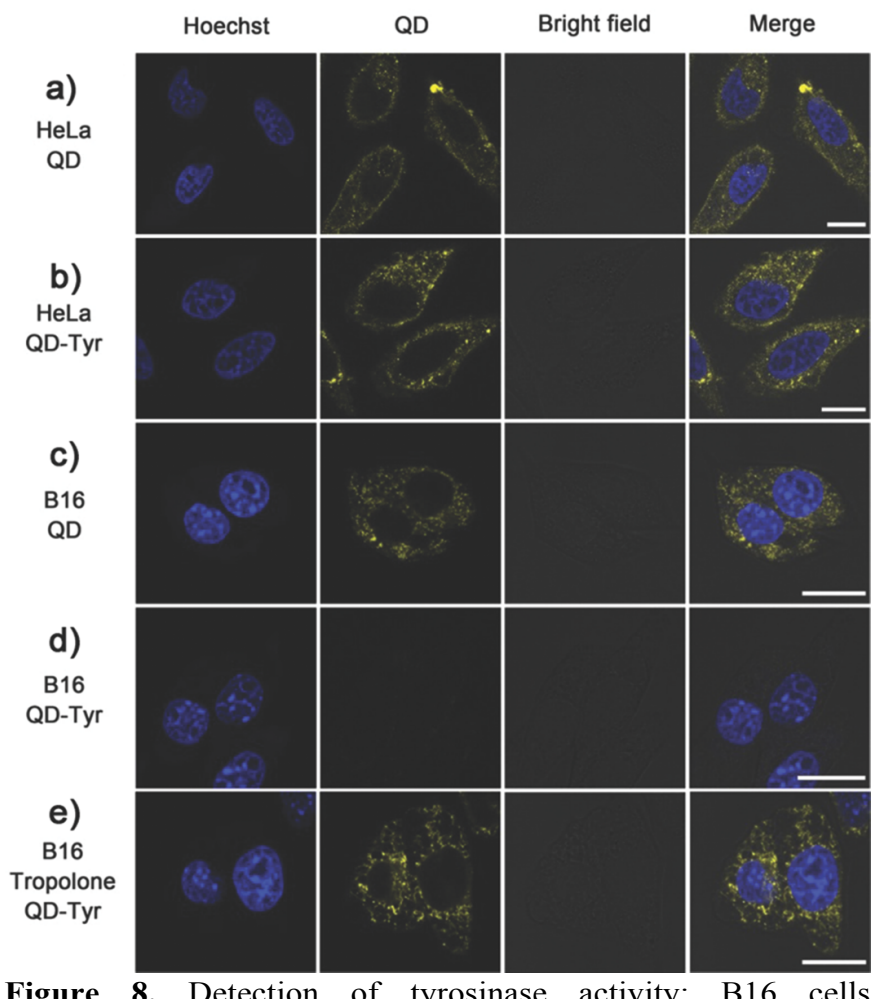

Figure 8. Detection of tyrosinase activity; B16 cells overexpressing tyrosinase show no emission when incubated with quantum dots modified with tyrosine (d); the emission is recovered in the presence of the tyrosinase inhibitor tropolone (e). Scale bars: $30 \mu \mathrm{m}$. Reprinted with permission from ${ }^{68}$. Copyright (2015) Willey-VCH.

\section{Intracellular detection of enzymes}

Several approaches have been proposed for detecting enzyme activity in live cells with photoluminescent nanosensors. For example quantum dots functionalised with tyrosine can be used to detect tyrosinase activity intracellularly. ${ }^{68}$ When tyrosinase oxidises tyrosine to dopachrome an electron transfer process is triggered that 
quenches the fluorescence emission of the quantum dots. With this strategy it is possible to differentiate B16 melanoma cells, which express high concentrations of tyrosinase, from $\mathrm{HeLa}$ cells with low tyrosinase expression rates. The B16 cells showed reduced fluorescence emission that could be recovered in the presence of tropolone, a reversible inhibitor of the enzyme (Figure 8).

Telomerase is a vital enzyme for DNA replication that has been found overexpressed in many cancesr. Telomerase activity can be detected inside cells with gold nanoparticles modified with nicked molecular beacons containing a shorter telomerase primer (TSP). ${ }^{69}$ The nicked molecular beacon is a DNA hairpin structure containing a fluorophore that is quenched by the gold nanoparticle in the absence of telomerase activity. When telomerase is present it elongates the TSP. This leads to a reorganisation of the $3 \mathrm{D}$ structure of the DNA probe in which the fluorophore is positioned far away from the quenching nanoparticle. Under this condition the fluorescence turns on, which enables detecting telomerase activity in live cells.

\section{Conclusions}

Nanosensors are enabling new strategies for intracellular detection that show many advantages compared to traditional probes. For example encapsulating organic dyes into a nanometric polymeric matrix may improve cell delivery and reduce toxic effects. ${ }^{47,70}$ The outstanding optical properties of quantum dots, ${ }^{30}$ upconverting nanocrystals ${ }^{41}$ and metallic nanoclusters, ${ }^{52}$ which are more photostable and afford higher signal-to-noise ratios than conventional fluorescent probes, are particularly interesting in bioimaging, especially in strategies that require long exposure times such as FLIM. ${ }^{30}$ However most of the strategies for intracellular detection devised so far are focused on the detection of physicochemical parameters such as $\mathrm{pH}$, redox potential, temperature or the concentration of ions. While measuring these parameters is crucial for understanding how cells work, cell regulation is mainly originated by the orchestrated action of regulating proteins (e.g. transcription factors, enzymatic cascades). Although the nanoflare technology enables detecting the levels of mRNA in real time, and therefore it is optimal to study mechanisms of cell regulation, proteins often suffer post-translational modifications that define their function and that cannot be detected at the RNA level. Therefore it would be desirable to fabricate nanosensors for the intracellular detection of proteins in real time and in live cells. When combined with nanosensors for the detection of mRNA, these new approaches could reveal mechanisms of cell regulation from a biomolecular perspective.

\section{Acknowledgements}

Financial support from Tenovus Scotland is acknowledged. Dr. de la Rica acknowledges a Tom West Analytical Fellowship from the Analytical Chemistry Trust Fund.

\section{Notes and references}

${ }^{a}$ Centre for Molecular Nanometrology, WestCHEM, Pure and Applied Chemistry, University of Strathclyde, 295 Cathedral Street, Glasgow G1 1XL, U.K.

* roberto.delarica@strath.ac.uk
H. Nakamura, K. Nakamura and J. Yodoi, Annu. Rev. Immunol., 1997, 15, 351-369.

C. Marchal and B. Miotto, J. Cell. Physiol., 2015, 230, 743-751.

L. Lu and W. Hong, Semin. Cell Dev. Biol., 2014, 31, 30-39.

D. M. Muoio, Cell, 2014, 159, 1253-1262.

J. A. Mindell, Annu. Rev. Physiol., 2012, 74, 69-86.

L. Rodríguez-Lorenzo, R. de la Rica, R. A. Álvarez-Puebla, L. M. Liz-Marzán and M. M. Stevens, Nat. Mater., 2012, 11, 604-607.

D. Cecchin, R. de la Rica, R. E. S. Bain, M. W. Finnis, M. M. Stevens and G. Battaglia, Nanoscale, 2014, 6, 9559-62.

L. Rodríguez-Lorenzo, R. a. Álvarez-Puebla, I. Pastoriza-Santos, S. Mazzucco, O. Stéphan, M. Kociak, L. M. Liz-Marzán and F. J. G. De Abajo, J. Am. Chem. Soc., 2009, 131, 4616-4618.

A. Crut, B. Géron-Landre, I. Bonnet, S. Bonneau, P. Desbiolles and C. Escudé, Nucleic Acids Res., 2005, 33, 1-9.

S. M. Stavis, J. B. Edel, K. T. Samiee and H. G. Craighead, Lab Chip, 2005, 5, 337-343.

K. Kobayashi, K. Niikura, C. Takeuchi, S. Sekiguchi, T. Ninomiya, K. Hagiwara, H. Mitomo, Y. Ito, Y. Osada and K. Ijiro, Chem. Commun. (Camb)., 2014, 50, 1265-1267.

X. Liu, Y. Chen, H. Li, N. Huang, Q. Jin, K. Ren and J. Ji, $A C S$ Nano, 2013, 7, 6244-6257.

K. Kettler, K. Veltman, D. van de Meent, A. van Wezel and a. J. Hendriks, Environ. Toxicol. Chem., 2014, 33, 481-492.

W. H. De Jong and P. J. a Borm, Int. J. Nanomedicine, 2008, 3, 133-149.

O. Agbulut, A. Huet, N. Niederländer, M. Puceat, P. Menasché and C. Coirault, J. Biol. Chem., 2007, 282, 10465-10471.

R. de la Rica, D. Aili and M. M. Stevens, Adv. Drug Deliv. Rev., 2012, 64, 967-978.

F. Zhao, Y. Zhao, Y. Liu, X. Chang, C. Chen and Y. Zhao, Small, 2011, 7, 1322-1337.

D. Nogueira, M. Mitjans, C. Rolim and M. Vinardell, Nanomaterials, 2014, 4, 454-484.

P. Rivera-Gil, D. Jimenez De Aberasturi, V. Wulf, B. Pelaz, P. Del Pino, Y. Zhao, J. M. De La Fuente, I. Ruiz De Larramendi, T. Rojo, X. J. Liang and W. J. Parak, Acc. Chem. Res., 2013, 46, 743749.

N. Lewinski, V. Colvin and R. Drezek, Small, 2008, 4, 26-49.
E. a. Vitol, Z. Orynbayeva, G. Friedman and Y. Gogotsi, J. Raman Spectrosc., 2012, 43, 817-827. 
J. R. Casey, S. Grinstein and J. Orlowski, Nat. Rev. Mol. Cell Biol., 2010, 11, 50-61.

M. J. Marín, F. Galindo, P. Thomas and D. a. Russell, Angew. Chemie - Int. Ed., 2012, 51, 9657-9661.

H. Izumi, T. Torigoe, H. Ishiguchi, H. Uramoto, Y. Yoshida, M. Tanabe, T. Ise, T. Murakami, T. Yoshida, M. Nomoto and K. Kohno, Cancer Treat. Rev., 2003, 29, 541-549.

V. M. Chauhan, G. R. Burnett and J. W. Aylott, Analyst, 2011, 136, 1799-1801.

J. Han and K. Burgess, Chem. Rev., 2010, 110, 2709-2728.

D. F. Baxter, M. Kirk, A. F. Garcia, A. Raimondi, M. H. Holmqvist, K. K. Flint, D. Bojanic, P. S. Distefano, R. Curtis and Y. Xie, J. Biomol. Screen. Off. J. Soc. Biomol. Screen., 2002, 7, 79-85.
R. Hu, M.-L. He, H. Hu, B.-X. Yuan, W.-J. Zang, C.-P. Lau, H.-F. Tse and G.-R. Li, J. Cell. Physiol., 2009, 220, 765-770.

D. E. Clapham, Cell, 1995, 80, 259-268.

M. Barbagallo, L. M. Resnick, L. J. Dominguez and G. Licata, Diabetes Metab., 1997, 23, 281-294.

A. R. Marks, Circulation, 2003, 107, 1456-1459.

D. Si, T. Epstein, Y. E. Koo Lee and R. Kopelman, Anal. Chem., 2012, 84, 978-986.

J. R. Henderson, D. a. Fulton, C. J. McNeil and P. Manning, Biosens. Bioelectron., 2009, 24, 3608-3614.

C. M. Lamy, O. Sallin, C. Loussert and J. Y. Chatton, ACS Nano, 2012, 6, 1176-1187.

S. Svenson and D. A. Tomalia, Adv. Drug Deliv. Rev., 2005, 57, 2106-2129.

J. M. Dubach, D. I. Harjes and H. A. Clark, Nano Lett., 2007, 7, 1827-1831.

K. Y. Pu, Z. Luo, K. Li, J. Xie and B. Liu, J. Phys. Chem. C, 2011, 115, 13069-13075.

R. de la Rica, L. W. Chow, C. Horejs , M. Mazo, C. Chiappini, E. T. Pashuck, R. Bitton and M. M. Stevens, Chem. Commun., 2014, 50, 10648-10650.

T. V O’Halloran, Science, 1993, 261, 715-725.

M. D. Pluth, E. Tomat and S. J. Lippard, Annu. Rev. Biochem., 2011, 80, 333-355.

M. P. Murphy, Biochem. J., 2009, 417, 1-13.

L. Sena and N. Chandel, Mol. Cell, 2012, 48, 158-167.

W. K. Oh, Y. S. Jeong, S. Kim and J. Jang, ACS Nano, 2012, 6, 8516-8524.

D. Lee, S. Khaja, J. C. Velasquez-Castano, M. Dasari, C. Sun, J. Petros, W. R. Taylor and N. Murthy, Nat. Mater., 2007, 6, 765769.

L. M. Uusitalo and N. Hempel, Int. J. Mol. Sci., 2012, 13, 10660 10679 .

R. Sakaguchi, S. Kiyonaka and Y. Mori, Curr. Opin. Biotechnol., 2014, 31C, 57-64.

K. Okabe, N. Inada, C. Gota, Y. Harada, T. Funatsu and S. Uchiyama, Nat. Commun., 2012, 3, 705.

G. Kucsko, P. C. Maurer, N. Y. Yao, M. Kubo, H. J. Noh, P. K. Lo, H. Park and M. D. Lukin, Nature, 2013, 500, 54-58.

J. S. Donner, S. a. Thompson, M. P. Kreuzer, G. Baffou and R. Quidant, Nano Lett., 2012, 12, 2107-2111. 
65 D. S. Seferos, D. A. Giljohann, H. D. Hill, A. E. Prigodich and C A. Mirkin, J. Am. Chem. Soc., 2007, 129, 15477-15479.

66 A. E. Prigodich, P. S. Randeria, W. E. Briley, N. J. Kim, W. L. Daniel, D. A. Giljohann and C. A. Mirkin, Anal. Chem., 2012, 84, 2062-2066.

67 S. Jiang and Y. Zhang, Langmuir, 2010, 26, 6689-6694.

68 X. Zhu, J. Hu, Z. Zhao, M. Sun, X. Chi, X. Wang and J. Gao, Small, 2015, 11, 862-870.

69 R. Qian, L. Ding, L. Yan, M. Lin and H. Ju, J. Am. Chem. Soc., 2014, 136, 8205-8208.

70 V. J. Hammond, J. W. Aylott, G. M. Greenway, P. Watts, A. Webster and C. Wiles, Analyst, 2008, 133, 71-75. 